\title{
Reconstructing and Forecasting the COVID-19 Epidemic in the US Using a 5-Parameter Logistic Growth Model
}

Ding-Geng Chen ( $\nabla$ dinchen@email.unc.edu )

University of North Carolina at Chapel Hill https://orcid.org/0000-0002-3199-8665

\section{Xinguang Chen}

University of Florida

Jenny Ke Chen

Cornell University

\section{Research}

Keywords: COVID-19, Logistic growth model, USA, Prediction, Reconstruction, Under-detection

Posted Date: May 14th, 2020

DOl: https://doi.org/10.21203/rs.3.rs-22956/v2

License: (c) (i) This work is licensed under a Creative Commons Attribution 4.0 International License.

Read Full License

Version of Record: A version of this preprint was published at Global Health Research and Policy on May 15th, 2020. See the published version at https://doi.org/10.1186/s41256-020-00152-5. 
4

112 Department of Epidemiology, University of Florida, USA;

12 email: jimax.chen@ufl.edu

$13{ }^{3}$ Department of Statistics and Data Science, Cornell University, USA;

14 email: jkc229@cornell.edu

15

16

17 Corresponding author: Ding-Geng Chen, University of North Carolina at Chapel Hill School of

18 Social Work, Tate-Turner Kuralt Building 548-C, CB \#3550, Chapel Hill, NC, 27599; email:

19 dinchen@email.unc.edu Using a 5-Parameter Logistic Growth Model

Ding-Geng Chen, $\mathrm{PhD}^{1}$, Xinguang Chen, $\mathrm{PhD}^{2}$, Jenny K. Chen, $\mathrm{BSc}^{3}$

${ }^{1}$ School of Social Work, University of North Carolina, USA; Department of Statistics, University of Pretoria, South Africa, email: dinchen@email.unc.edu

20

21

22

23 
Abstract

Background: Many studies have modeled and predicted the spread of COVID-19 (coronavirus disease 2019) in the U.S. using data that begins with the first reported cases. However, the

27 shortage of testing services to detect infected persons makes this approach subject to error due to its underdetection of early cases in the U.S. Our new approach overcomes this limitation and provides data supporting the public policy decisions intended to combat the spread of COVID-19 epidemic.

Methods: We used Centers for Disease Control and Prevention data documenting the daily new and cumulative cases of confirmed COVID-19 in the U.S. from January 22 to April 6, 2020, and reconstructed the epidemic using a 5-parameter logistic growth model. We fitted our model to data from a 2-week window (i.e., from March 21 to April 4, approximately one incubation period) during which large-scale testing was being conducted. With parameters obtained from this modeling, we reconstructed and predicted the growth of the epidemic and evaluated the extent and potential effects of underdetection.

Results: The data fit the model satisfactorily. The estimated daily growth rate was $16.8 \%$ overall with 95\% CI: [15.95\%, 17.76\%], suggesting a doubling period of 4 days. Based on the modeling result, the tipping point at which new cases will begin to decline will be on April $7^{\text {th }}, 2020$, with a peak of 32,860 new cases on that day. By the end of the epidemic, at least 792,548 (95\% CI: $[789,162,795,934])$ will be infected in the U.S. Based on our model, a total of 12,029 cases were

43 not detected between January 22 (when the first case was detected in the U.S.) and April 4.

44 Conclusions: Our findings demonstrate the utility of a 5-parameter logistic growth model with 45 reliable data that comes from a specified period during which governmental interventions were 
46 appropriately implemented. Beyond informing public health decision-making, our model adds a

47 tool for more faithfully capturing the spread of the COVID-19 epidemic.

48 Keywords: COVID-19; epidemics; disease dynamics; population-based model; logistic growth

49 model; prediction; reconstruction; under-detection; tipping point; USA

50 List of Abbreviations: CDC (Centers for Disease Control and Prevention); CI (confidence

51 interval); COVID-19 (coronavirus disease 2019); SE (standard error)

52 


\section{Introduction}

Coronavirus disease 2019 (COVID-19) is an infection caused by a novel pathogen named SARS-Cov-2. Spreading worldwide in less than five months, the COVID-19 pandemic is a typical example of a global health issue. ${ }^{1}$ In the months since the first COVID-19 case was reported in the United States on January 22, 2020, many studies have employed different models to reconstruct the epidemic (i.e., the spread of COVID-19 within the United States only) and forecast its future trends, from simple growth models to classic susceptible-infected-recovered models. ${ }^{2}$ Yet due to the scarcity of available information about the early period of the COVID-19 epidemic, researchers lack sufficient data to construct complex and classic epidemiological models. In this context, the population-based ecological growth model is the preferable option for predicting the epidemic's future trajectory.

Researchers have developed various population-based models for modeling population dynamics and disease epidemics. One such model is the 1-parameter exponential growth model. In this model, population growth has no upper limit and is determined by one parameter of growth rate. To account for the upper limit of population growth, the 2-parameter logistic growth model was developed. In this model, the population growth rate is exponential in the beginning, but this growth rate gets smaller and smaller as population size approaches a maximum carrying capacity as detailed described in Richards ${ }^{3}$, McIntosh $^{4}$, Renshaw $^{5}$, Kingsland $^{6}$, and Vandermeer. ${ }^{7}$ To account for additional key characteristics of population growth, the 2-parameter logistic growth model has since been extended to 3-parameter, 4-parameter, and 5-parameter logistic growth models. These models have been widely used in other fields of research, including demography and analytical chemistry. ${ }^{8,9}$ Despite the many analytical advantages of these models, to our knowledge, no study has employed this 5-parameter logistic growth model 
to examine the COVID-19 epidemic in the United States or in other countries. Thus, one purpose

77 of this study is to assess the utility of the 5-parameter growth model in studying the dynamics of

78 the spread of COVID-19.

79 Unlike typical population growth models (in which the initial population is a known

80

81

82

83

84

85

86

87

88

89

90

91

\section{Data}

93

94

95

96

97

98 quantity), only a small number of COVID-19 cases were detected during the early phase of the epidemic in the United States. In all contexts, more extensive testing services detect more cases; when the initial time of an epidemic's outbreak is known, extensive testing can yield data that more accurately reflects the true growth of the epidemic. Data indicate that the incubation period of COVID-19 is about 14 days $^{10}$, and COVID-19 testing services in the U.S. became available in mid-March and were sustained thereafter following CDC guidelines. Therefore, the 14-day interval following the widespread implementation of testing should demonstrate the highest level of detection rates unaffected by the removal of infected individuals from the growth curve, presenting ideal data for model building. In principle, a model built with this data would more accurately capture and predict the growth of COVID-19 than models constructed from infection data ranging from the first detected case to the present.

\section{Methods}

Data for this study were the daily cumulative cases of COVID-19 in the U.S. from January 22 to April 6, 2020. This real-time data were compiled by the Centers for Disease Control and Prevention (CDC) and made available on their website at the time we conducted our study. ${ }^{11}$

\section{Models}

We modeled the data using the 5-parameter logistic growth model as below: 


$$
C(t)=C_{\min }+\frac{C_{\max }-C_{\min }}{\left[1+e^{-r\left(t-t_{\operatorname{mid}}\right)}\right]^{\alpha}}
$$

117 deviation of $\sigma$.

\section{Implementation of Modeling Analysis}

where

$$
1 / 23 / 2020, \ldots, 4 / 6 / 2020)
$$

4) $r$ is the daily exponential growth rate; and then to decrease; and of the model:

1) $C(t)$ is the number of cumulative cases of COVID-19 over time, $t(t=1 / 22 / 2020$,

2) $C_{\min }$ is the minimum number of cases at the beginning of the epidemic on January 22, 2020, when the first case was reported in the U.S.;

3) $C_{\max }$ is the maximum number of people infected by the time the epidemic ends (i.e. the model-predicted total number of Americans who will be infected with COVID-19);

5) $t_{\text {mid }}$ is the estimated tipping point when the number of new daily cases begins to level off

6) $\alpha$ is an asymmetric parameter quantifying the skewness of the distribution of daily new cases. $\alpha=1$ indicates a symmetric distribution centered at $t_{m i d} ; \alpha>1$ indicates faster increases in new cases before $t_{\text {mid }}$ and slower after $t_{\text {mid }}$; and the reverse if $\alpha<1$.

With Model 1 defined above, daily new cases $D(t)$ can be obtained by taking the first derivative

$$
D(t)=C^{\prime}(t)=\frac{\alpha r\left(C_{\text {max }}-C_{\text {min }}\right)}{\left[1+e^{-r\left(t-t_{\text {mid }}\right)}\right]^{\alpha+1}} \times e^{-r\left(t-t_{\text {mid }}\right)}+\in(t),
$$

where the error term $\in(t)$ is assumed to be normally distributed with mean 0 and standard

$$
\text { We conducted our data analysis using the software R. A 5-parameter logistic growth }
$$
model was fitted to the data for new daily infections from March 21, 2020 to April 4, 2020, as 
121 shown in Model 2. Using the R function “optim," we implemented modeling analysis using a

122 nonlinear optimization algorithm to minimize the sum of squared errors between the observed

123 and model-estimated data. The optimization process yielded estimates for the five parameters

$124 C_{\min }, C_{\text {max }}, t_{\text {mid }}, r$, and $\alpha$ with a significance level set at $p<0.05$ (two-sided).

125 With these five estimated model parameters, we estimated model-based cumulative cases

126 (using Model 1) and new cases (using Model 2) for each day from March 21 to April 4 and made

127 predictions about cumulative and new daily cases after April 4. We calculated the underdetection

128 of cases in this 2-week window by measuring the differences between the reported number and

129 the model-predicted number of cases.

Results

131

Model 2 fitted the observed cumulative daily cases from March 21 to April 4

132 satisfactorily and the model fit converged nicely. Table 1 summarizes the estimated parameters,

133 their standard error (SE), and their 95\% confidence intervals (CI). Except for $C_{\text {min }}$, all model

134 parameters were statistically significant at $p<0.001$ level. The lack of significance for

$135 C_{\min }$ appears to be reasonable given the small scale of this number relative to the other

136 parameters and the practical difficulties of determining the number of actual cases at the

137 beginning of the epidemic when the first few COVID-19 cases were detected and reported.

Table 1. Summary of parameter estimation

\begin{tabular}{|cccccc|}
\hline Parameter & Estimate & SE & p-value & Lower 95\% CI & Upper 95\% CI \\
\hline $\boldsymbol{C}_{\text {min }}$ & 29.999 & 2059.86 & 0.988 & -4007.33 & 4067.32 \\
\hline $\boldsymbol{C}_{\max }$ & 792,548 & 1727.56 & $<0.0001$ & 789,162 & 795,934 \\
\hline
\end{tabular}




\begin{tabular}{|cccccc|}
\hline $\boldsymbol{t}_{\text {mid }}$ & 76.9 & 0.456 & $<0.0001$ & 75.952 & 77.739 \\
\hline $\boldsymbol{r}$ & 0.16854 & 0.00463 & $<0.0001$ & 0.15947 & 0.17761 \\
\hline $\boldsymbol{\alpha}$ & 0.95364 & 0.06194 & $<0.0001$ & 0.83224 & 1.07504 \\
\hline
\end{tabular}

Note: Parameters were estimated based on daily cases of COVID-19 in the U.S. between March 21, 2020 and April 4, 2020.

Based on our model estimates, at least 792,548 (95\% CI: [789,162, 795,934]) Americans will have been infected with COVID-19 by the time the epidemic ends. This number is slightly more than twice the number of infections that had occurred in the U.S. by April 6. For reasons we discuss later, this estimate may be conservative, as the total number of reported cases exceeded 800,000 on April 21, as we completed our revisions of this paper.

Our estimated tipping point for new daily cases was on about April 7, 77 days (95\% CI: $[76,78])$ from the beginning of the epidemic on January 22. In other words, our model predicted that the epidemic curve in the U.S. would begin to flatten around April 6-8, 2020. This estimation corroborates recent reporting that new daily cases in the U.S. have remained somewhat constant beginning in early April. ${ }^{12}$ This tipping point suggests that it will take three to four more COVID-19 incubation periods (i.e., 6 to 8 weeks) for the U.S. to bring the epidemic under control, given our documentation and analysis of this process in China. ${ }^{10,13}$

The estimated exponential daily growth rate of COVID-19 in the U.S. population is $16.9 \%$ (95\% CI: $[15.9 \%, 17.8 \%])$, nearly the rate observed in China $(17.12 \%) .{ }^{10}$ This U.S. rate suggests that the number of total COVID-19 cases in the U.S. will double every four days if no anti-epidemic actions are in place. The estimated asymmetric parameter $\alpha$ was 0.954 (95\% CI: $[0.832,1.075])$, which is not statistically different than $\alpha=1.0$. This result indicates that changes in COVID-19 cases before and after the predicted tipping point of April 7 will follow a similar pattern. 
For further illustration, Table 2 summarizes three sets of information ordered by days

163 from the beginning of the epidemic: the data used for the model fitting section, a smaller

164 reconstruction section, and a prediction section. Our fitted model detected substantial

165 underdetected COVID-19 cases. By April 7, when this study was completed, the CDC reported a

166 total of 395,011 detected cases; with our model, we predicted that CDC data for reported cases in

167 fact underreported about 19,291 cases up to April 9.

168

Using a 2-week interval (i.e., March 21 to April 4) of data, our model's prediction of the

number of new daily cases from April 5 to April 11 matched quite well with the observed data.

170 For example, the model-predicted number on April 9 was 31,705, very close to the observed

171 number of 31,582 .

These results should be interpreted with caution. The estimated sum square of error $\hat{\sigma}=$

1732638.434 is quite large, meaning that although our model fitted the 2 -week interval of data very

174 well, a large amount of variation in the data is not explained by this model.

175

176

177

Table 2. Illustration of data usage with reported, predicted, and underreported counts

\begin{tabular}{|c|c|c|c|c|c|c|c|}
\hline \multirow[t]{2}{*}{ Data Usage } & \multirow[t]{2}{*}{ Days } & \multirow[t]{2}{*}{ Date } & \multicolumn{2}{|c|}{ Reported Cases } & \multicolumn{2}{|c|}{ Predicted } & \multirow{2}{*}{$\begin{array}{l}\text { Under- } \\
\text { reported }\end{array}$} \\
\hline & & & Total & Daily & Daily & Total & \\
\hline \multirow[t]{6}{*}{ Reconstruction } & 54 & $3 / 15 / 2020$ & 3487 & 1253 & 3108 & 19781 & 16294 \\
\hline & 55 & $3 / 16 / 2020$ & 4226 & 739 & 3623 & 23141 & 18915 \\
\hline & 56 & $3 / 17 / 2020$ & 7038 & 2812 & 4218 & 27054 & 20016 \\
\hline & 57 & $3 / 18 / 2020$ & 10442 & 3404 & 4902 & 31606 & 21164 \\
\hline & 58 & $3 / 19 / 2020$ & 15219 & 4777 & 5687 & 36892 & 21673 \\
\hline & 59 & $3 / 20 / 2020$ & 18747 & 3528 & 6584 & 43019 & 24272 \\
\hline \multirow[t]{11}{*}{ Fitting } & 60 & $3 / 21 / 2020$ & 24583 & 5836 & 7603 & 50102 & 25519 \\
\hline & 61 & $3 / 22 / 2020$ & 33404 & 8821 & 8755 & 58269 & 24865 \\
\hline & 62 & $3 / 23 / 2020$ & 44183 & 10779 & 10047 & 67658 & 23475 \\
\hline & 63 & $3 / 24 / 2020$ & 54453 & 10270 & 11485 & 78411 & 23958 \\
\hline & 64 & $3 / 25 / 2020$ & 68440 & 13987 & 13070 & 90676 & 22236 \\
\hline & 65 & $3 / 26 / 2020$ & 85356 & 16916 & 14797 & 104598 & 19242 \\
\hline & 66 & $3 / 27 / 2020$ & 103321 & 17965 & 16656 & 120315 & 16994 \\
\hline & 67 & $3 / 28 / 2020$ & 122653 & 19332 & 18624 & 137947 & 15294 \\
\hline & 68 & $3 / 29 / 2020$ & 140904 & 18251 & 20670 & 157589 & 16685 \\
\hline & 69 & $3 / 30 / 2020$ & 163539 & 22635 & 22750 & 179298 & 15759 \\
\hline & 70 & $3 / 31 / 2020$ & 186101 & 22562 & 24810 & 203082 & 16981 \\
\hline
\end{tabular}




\begin{tabular}{|lccccccc|}
\hline & $\mathbf{7 1}$ & $\mathbf{4 / 1 / 2 0 2 0}$ & $\mathbf{2 1 3 1 4 4}$ & $\mathbf{2 7 0 4 3}$ & $\mathbf{2 6 7 8 4}$ & $\mathbf{2 2 8 8 8 9}$ & $\mathbf{1 5 7 4 5}$ \\
\cline { 2 - 7 } & $\mathbf{7 2}$ & $\mathbf{4 / 2 / 2 0 2 0}$ & $\mathbf{2 3 9 2 7 9}$ & $\mathbf{2 6 1 3 5}$ & $\mathbf{2 8 6 0 0}$ & $\mathbf{2 5 6 5 9 7}$ & $\mathbf{1 7 3 1 8}$ \\
\cline { 2 - 7 } & $\mathbf{7 3}$ & $\mathbf{4 / 3 / 2 0 2 0}$ & $\mathbf{2 7 7 2 0 5}$ & $\mathbf{3 7 9 2 6}$ & $\mathbf{3 0 1 8 0}$ & $\mathbf{2 8 6 0 1 0}$ & $\mathbf{8 8 0 5}$ \\
\hline \multirow{6}{*}{ Forecast } & $\mathbf{7 4}$ & $\mathbf{4 / 4 / 2 0 2 0}$ & $\mathbf{3 0 4 8 2 6}$ & $\mathbf{2 7 6 2 1}$ & $\mathbf{3 1 4 5 3}$ & $\mathbf{3 1 6 8 5 5}$ & $\mathbf{1 2 0 2 9}$ \\
& 75 & $4 / 5 / 2020$ & 330891 & 26065 & 32352 & 348791 & 17900 \\
& 76 & $4 / 6 / 2020$ & 374329 & 43438 & 32830 & 381419 & 7090 \\
& 47 & $4 / 7 / 2020$ & 395011 & 20682 & 32860 & 414302 & 19291 \\
& $4 / 8 / 2020$ & 427460 & 32449 & 32436 & 446987 & 19527 \\
& 78 & $4 / 9 / 2020$ & 459165 & 31705 & 31582 & 479030 & 19865 \\
\hline 79 & $4 / 10 / 2020$ & 492416 & 33251 & 30340 & 510021 & 17605 \\
\hline
\end{tabular}

178

179

180

181

182

183

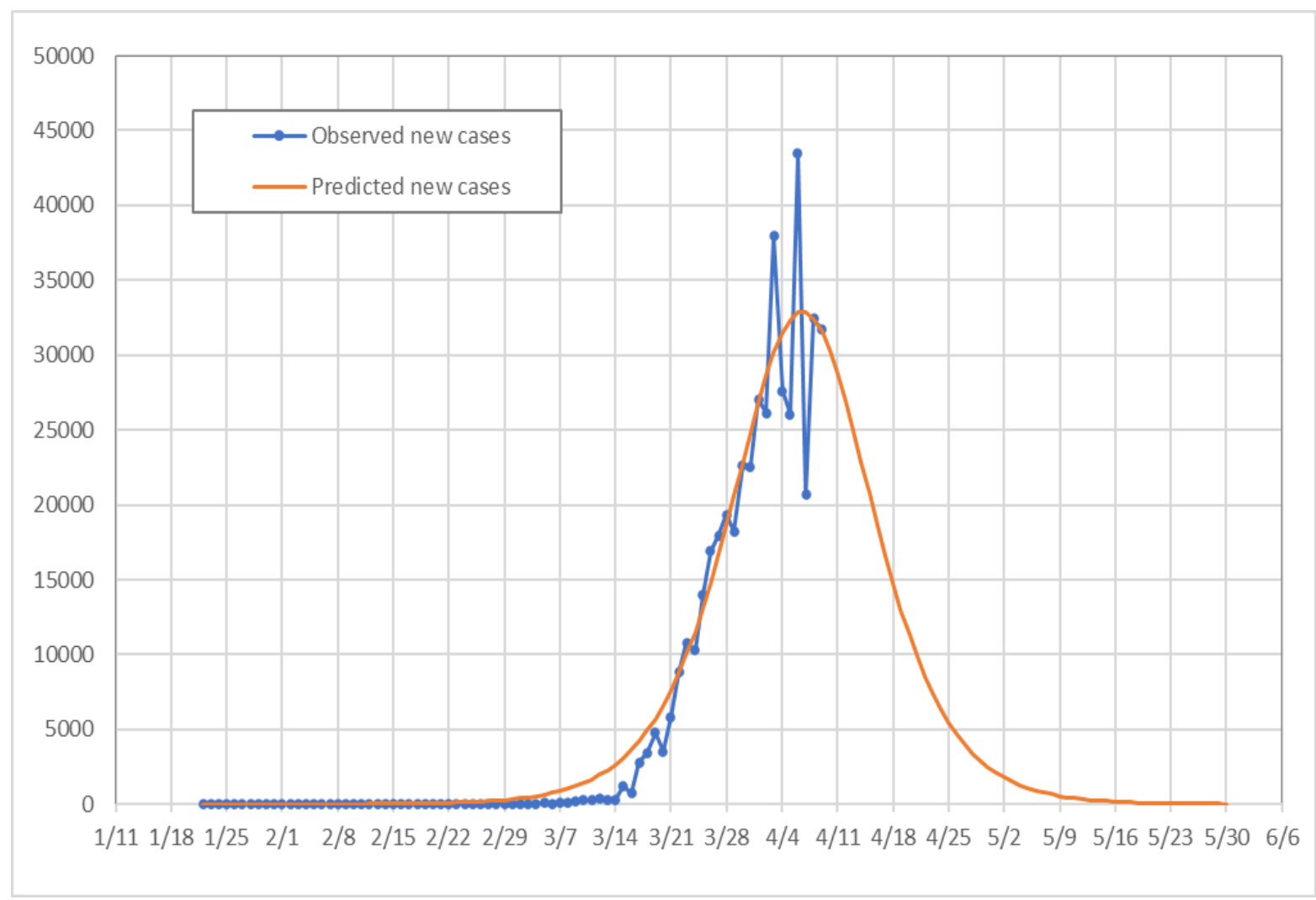

184

Below we provide two figures comparing the observed and model-predicted dynamics of new daily cases (Figure 1) and of cumulative cases (Figure 2). Overall, the model we constructed from only two weeks of data very closely predicted the reported numbers of both new and cumulative cases. Correspondingly, our model predicts that the cumulative cases will continue to increase rapidly after the tipping point until early May, as illustrated in Figure 2.

Figure 1. Observed vs. model-estimated and forecasted daily new COVID-19 cases, January 22 May 30, U.S.A.

10 | P a g e 


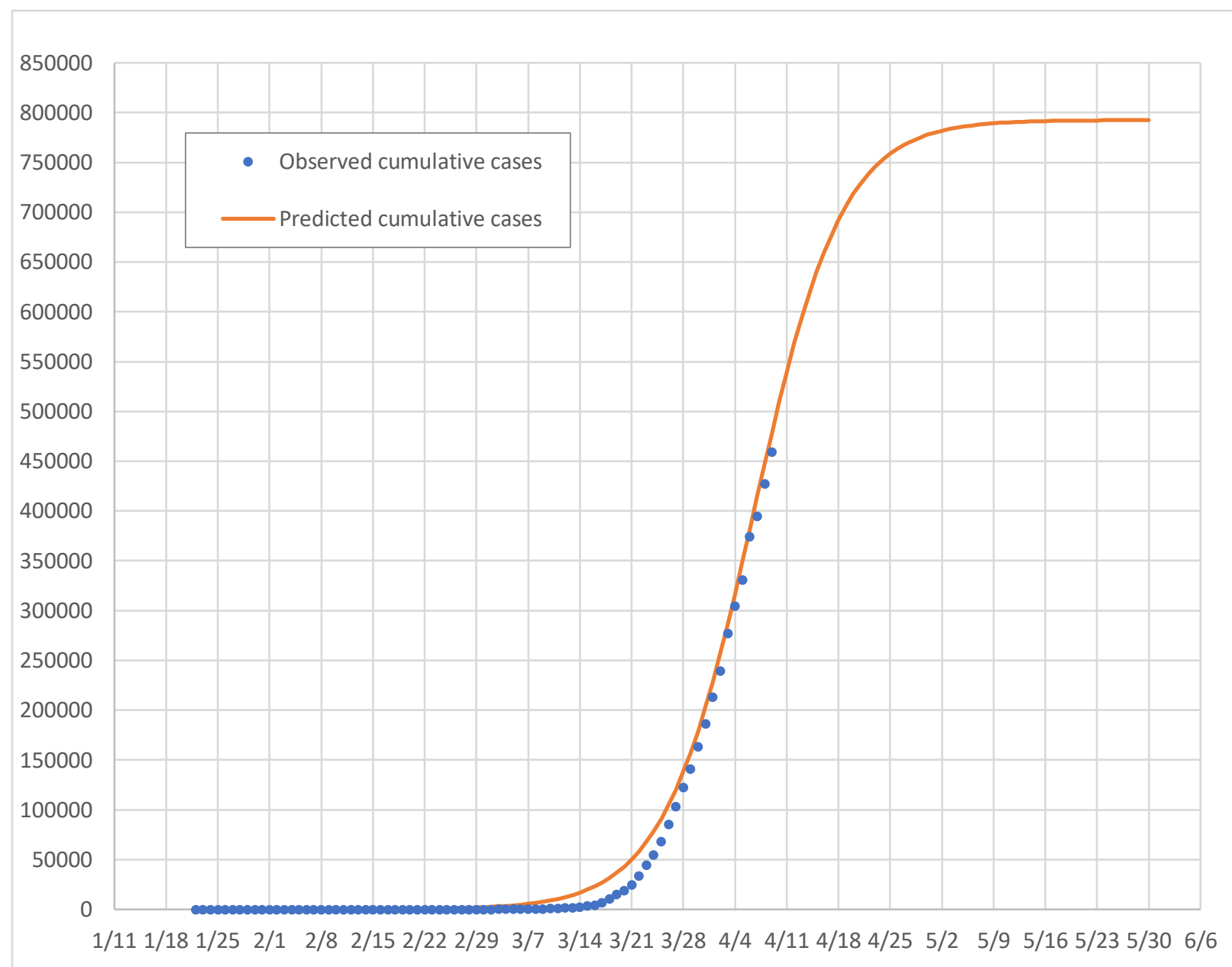

Figure 2. Observed vs. model-estimated and forecasted daily cumulative COVID-19 cases, January 22 - May 30, U.S.A.

\section{Discussion}

This study details our efforts to model, reconstruct, and forecast the COVID-19 epidemic using a 5-parameter logistic growth model - a method widely used in demography, biology, and other hard sciences. To our knowledge, we are the first to use this model to analyze the COVID19 epidemic in the U.S. We also developed and used our model through an innovative approach.

Namely, to fit the model we intentionally used data from a 2-week period when new cases could be more completely detected, and we then used this fitted model to reconstruct the growth of 
cases before and after the 2-week period as well as to forecast the future development of the epidemic beyond the study period.

Based on findings from our modeling analysis, there is not a high likelihood that the number of daily new cases will increase continuously after the tipping point (i.e., April 7, 2020). However, our model's estimation that at least 800,000 Americans will be infected over the course of the epidemic may be conservative, given that the total number of reported cases exceeded 800,000 on April 21, as we completed our revisions of this paper, while the new cases fluctuated between 26,000 and 35,000 per day due to the increased appearance of cases in other cities and states outside of New York.

This conservative estimation is potentially attributable to three factors. First, the exponential growth of our logistic model is very sensitive to differences in growth rate, and a small difference in the number of early cases can lead to a sizeable difference in predictions of subsequent cases. Second, although we strategically selected a 2-week interval of data that we believed would yield the best model for predicting the epidemic's growth, this data likely still underreported the actual number of COVID-19 infections, making our estimated growth rate smaller than the true growth rate. For example, the estimated exponential growth rate of COVID19 is $17.12 \%$ for China $^{10}$, higher than $16.85 \%$, the rate we estimated for the U.S. A small difference in the exponential growth rate can result in substantial differences in the maximum number of infections. And third, the data used for this analysis is from March 21 to April 4, 2020, where most of the reported cases are from the states of New York and New Jersey. The reported cases from these two states are flattened from reported CDC. Still, more cases are reported from other states, especially from the states of Michigan, Florida, Louisiana, which would add to the cases from New York and New Jersey to exceed the 800,000 predicted. 
The accuracy of our model is also contingent on the federal- and state-level policy

222

223

224

225

226

227

228

229

230

231

232

233

234

235

236

237

238

239

240

241

242

243

decisions that emerge in coming months. Although many states have implemented strict shelterin-place policies to slow down the epidemic's spread, several states still have no such policies in place. In the absence of further policy action, we expect that more cases will be reported which may greatly surpass the estimated 800,000 , and that the actual infection tipping point may occur later in April. Indeed, significant variations still persist in the estimated total infections in the U.S. even in light of available data: Ferguson et al. ${ }^{14}$ predicted 2.2 million cases whereas the CDC's worst-case scenario model predicted a shocking 214 million cases. ${ }^{15}$ At this moment, it remains unclear which estimates are more reliable. The accuracy of our estimation will be tested in light of emerging data on the progression of the epidemic in the United States.

The daily exponential growth rate of COVID-19 is $16.85 \%$ for the U.S. population, nearly the rate observed in China (17.12\%). ${ }^{10}$ Daily exponential growth rates can be obtained with limited data in the early period of an epidemic, and they provide a dynamic measure of instantaneous change, making doubling times calculated based on growth rate highly useful for directing and evaluating anti-epidemic measures. The U.S.'s daily exponential growth rate suggests that the number of COVID-19 infections will double every four days. For example, if the total cases are 500,000 today, there will be 1,000,000 in four days (with 40,000 anticipated deaths) if no timely anti-epidemic measures are implemented. No one - including policymakers, medical and health professionals, and the general public - should ignore this evidence of the pressing need to control the pandemic.

\section{Conclusion}

Understanding and curbing the COVID-19 epidemic in the U.S. is an essential part of fighting the pandemic globally. ${ }^{1}$ This study provides data important for informing public health 
244 decision-making designed to end the epidemic in the U.S. Our study also demonstrates the utility

245 and efficiency of the 5-parameter logistic growth model for examining the dynamics of an

246 epidemic in its early period when little data is available. Additionally, our selection of the 5-

247 parameter logistic exponential growth model was based on intensive testing of other models,

248 including 2-parameter, 3-parameter, and 4-parameter models. Of all models tested, the 5-

249 parameter produced the most accurate results and generated key information, including the

250 exponential growth rate, the doubling time for the epidemic, and the tipping point when daily

251 new cases will level off.

252 Our study's findings should be considered in light of their limitations. First, our strategic

253 selection of data from a specific timeframe is more subjective than objective, and not applicable

254 in all contexts. Researchers applying this method in different countries/regions with different

255 anti-epidemic strategies implemented in different ways should make their own determinations

256 regarding the optimal timeframe to select for their modeling. We selected the 2-week interval

257 from March 21 to April 4 because this interval spans approximately one COVID-19 incubation

258 period and because the U.S. government began implementing widespread testing services by the

259 beginning of this period, meaning that data from this interval potentially captured a more

260 representative set of new cases. Interested readers can conduct their own analyses using this

261 model while expanding on this time window to further assess the utility of this method. So far,

262 the model's short-term predicted daily cases are quite close to the observed daily cases, as shown

263 by Table 2 . However, our model's long-term predictions of future new daily cases may not be

264 accurate (which is true of any model-based long-term prediction), so these long-term predictions

265 should be considered with caution. 
Second, additional work is needed to improve confidence in the accuracy $C_{\text {min }}$, the

267 minimum number of cases at the beginning of an epidemic. It is challenging to improve this

268 estimation given the large range of different measures in the model. For example, the range

269 between $C_{\min }$ and $C_{\max }$ in our analysis is from about 30 to about 800,000 . Furthermore, the

270 number of reported cases at the beginning of the epidemic is highly unreliable due to a lack of

271 testing protocols and perhaps a lack of awareness of the incipient epidemic itself, which will lead

272 in turn to an unreliable estimation of $C_{\min }$.

273 Despite the limitations, findings from this study provide timely data that can inform

274 public health decision-making and policies designed to end the epidemic. We will continue to

275 update our model as more data become available and the COVID-19 epidemic in the United

276 States continues to evolve.

277

278 Acknowledgments: We appreciate Dr. Jordan Wingate for his professional editing for this

279 manuscript, which substantially improves the quality of this manuscript. We also appreciate the

280 two anonymous reviewers for their comments and suggestions to make this manuscript

281 publishable.

282

\section{Declarations}

284 Ethics approval and consent to participate

285 Not applicable.

286 Consent for publication

287 Not applicable.

288 Availability of data and materials 
289 The dataset supporting the conclusions of this article is available from the Centers for Disease

290 Control website,

291 https://www.cdc.gov/coronavirus/2019-ncov/cases-updates/cases-in-us.html.

292 Competing interests

293 The authors declare no competing interests associated with this study.

294 Funding

295 Not applicable.

296 Authors' contributions

297 All three authors participated in data validation, data analysis, and manuscript preparation.

298 Acknowledgements

299 Not applicable.

300 Authors' information (optional)

301 Dr. Ding-Geng Chen is the Wallace H. Kuralt distinguished professor in the School of Social

302 Work, University of North Carolina at Chapel Hill. Dr. Xinguang Chen is a professor in the 303 Department of Epidemiology, University of Florida. Ms. Jenny K. Chen is a undergraduate 304 student in the Department of Statistics and Data Science at Cornell University.

305

306

307

References

308 1. Chen X, Li H, Lucero-Prisno D, Abdullah A, Huang J, Laurence C, et al. What is global

309 health? Key concepts and clarification of misperceptions: report of the 2019 GHRP editorial 310 meeting. Glob Health Res Policy. 2020;5(14). doi:10.1186/s41256-020-00142-7 
311 2. Huang Y, Yang L, Dai H, Tian F, Chen K. Epidemic situation and forecasting of COVID-19

312 in and outside China. Bull World Health Organ. 2020; Mar 16. doi:10.2471/BLT.20.255158.

313 [Epub ahead of print]

314 3. Richards FJ. A flexible growth function for empirical use. J Exp Bot. 1959;10(2):290-301.

315 doi:10.1093/jxb/10.2.290

316 4. McIntosh RP. The background of ecology. New York: Cambridge University Press; 1985.

317 5. Renshaw E. Modeling biological populations in space and time. New York: Cambridge

318 University Press, 1991.

319 6. Kingsland SE. Modeling nature: episodes in the history of population ecology. Chicago:

320 University of Chicago Press; 1995.

321 7. Vandermeer J. How populations grow: the exponential and logistic equations. Nature

322 Education Knowledge [Internet]. 2010 [cited 2020 Apr 23];3(10):15. Available from:

323 https://www.nature.com/scitable/knowledge/library/how-populations-grow-the-exponential-and324 logistic-13240157/

325 8. Gottschalk PG, Dunn JR. The five-parameter logistic: a characterization and comparison with 326 the four-parameter logistic. Anal Biochem. 2005;343(1):54-65. doi:10.1016/j.ab.2005.04.035

327 9. Motulsky HJ, Brown RE. Assessing the (a)symmetry of concentration-effect curves: empirical 328 versus mechanistic models. BMC Bioinformatics. 2006;7:123.

329 10. Chen X, Yu B. First two months of the 2019 coronavirus disease (COVID-19) epidemic in 330 China: real-time surveillance and evaluation with a second derivative model. Glob Health Res 331 Policy. 2020;5(7). doi:10.1186/s41256-020-00137-4 
332 11. Centers for Disease Control and Prevention. Cases of coronavirus disease (COVID-19) in the 333 U.S. [Internet]. 2020 [cited 2020, Apr 7]. Available from:

334 https://www.cdc.gov/coronavirus/2019-ncov/cases-updates/cases-in-us.html

335 12. Johns Hopkins University and Medicine. New cases of COVID-19 in world countries

336 [Internet]. 2020 [cited 2020, Apr 22]. Available from: https://coronavirus.jhu.edu/data/new-cases

337 13. Chen X, Yu B, Chen D. Three months of COVID-19 in China: surveillance, evaluation, and 338 forecast from outbreak to control with a second derivative model. 2020. Submitted.

339 14. Ferguson NL, Laydon D, Nedjati-Gilani G, Imai N, Ainslie K, Baguelin M., et al. Impact of 340 non-pharmaceutical interventions (NPIs) to reduce COVID-19 mortality and healthcare demand.

341 WHO Collaborating Centre for Infectious Disease Modelling, MRC Centre for Global Infectious

342 Disease Analysis, Abdul Latif Jameel Institute for Disease and Emergency Analytics, Imperial

343 College London. 2020. https://www.imperial.ac.uk/media/imperial-

344 college/medicine/sph/ide/gida-fellowships/Imperial-College-COVID19-NPI-modelling-16-03-

345 2020.pdf. Accessed 12 Apr 2020.

346 15. Danner, C. CDC's worst-case coronavirus model: 214 million infected, 1.7 million dead.

347 New York Intelligencer [Internet]. 2020 Mar 13 [cited 2020, Apr 12]; Available from:

348 https://nymag.com/intelligencer/2020/03/cdcs-worst-case-coronavirus-model-210m-infected-1-

$349 \quad 7 \mathrm{~m}$-dead.html 
Figures

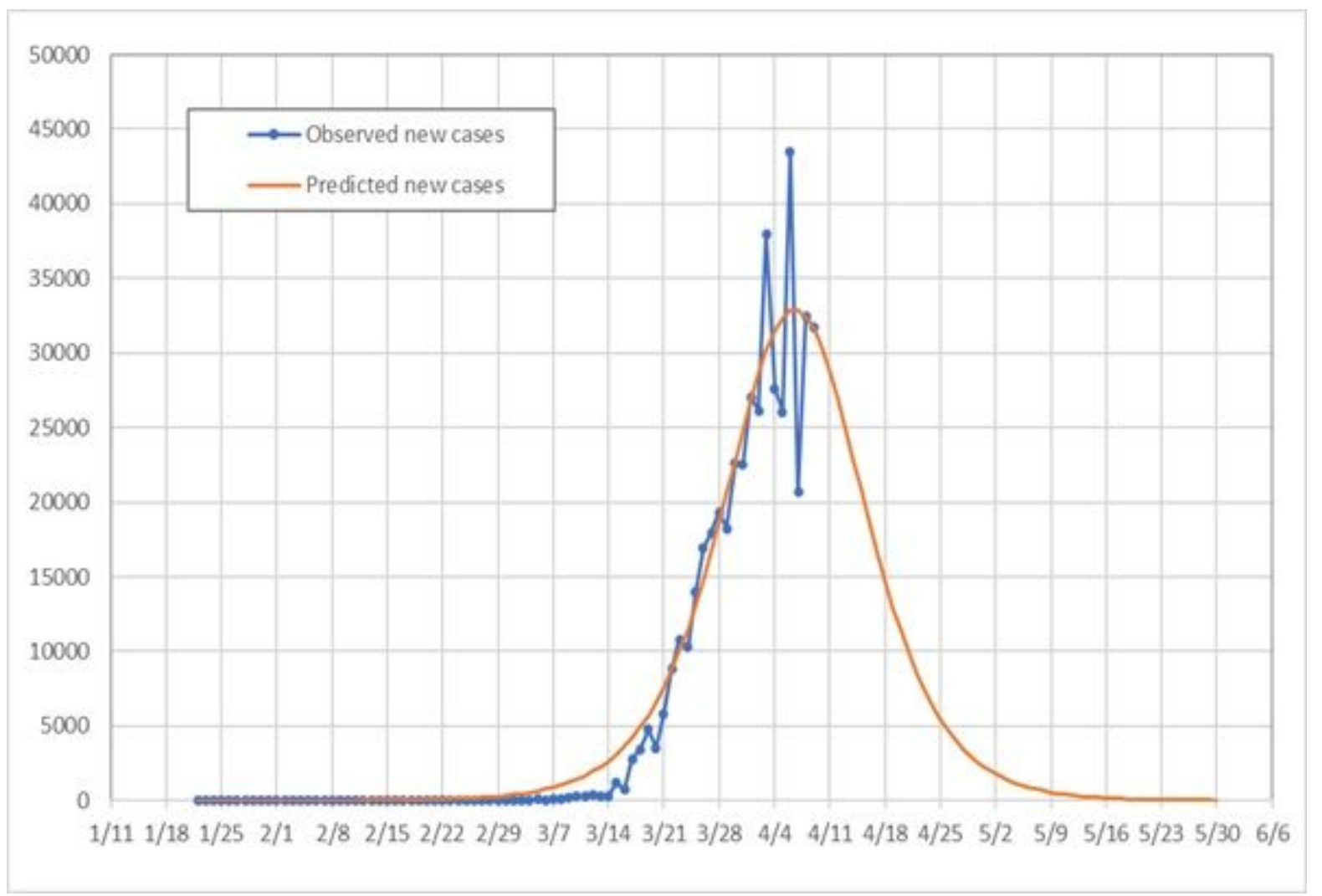

Figure 1

Observed vs. model-estimated and forecasted daily new COVID-19 cases, January 22 - May 30, U.S.A. 


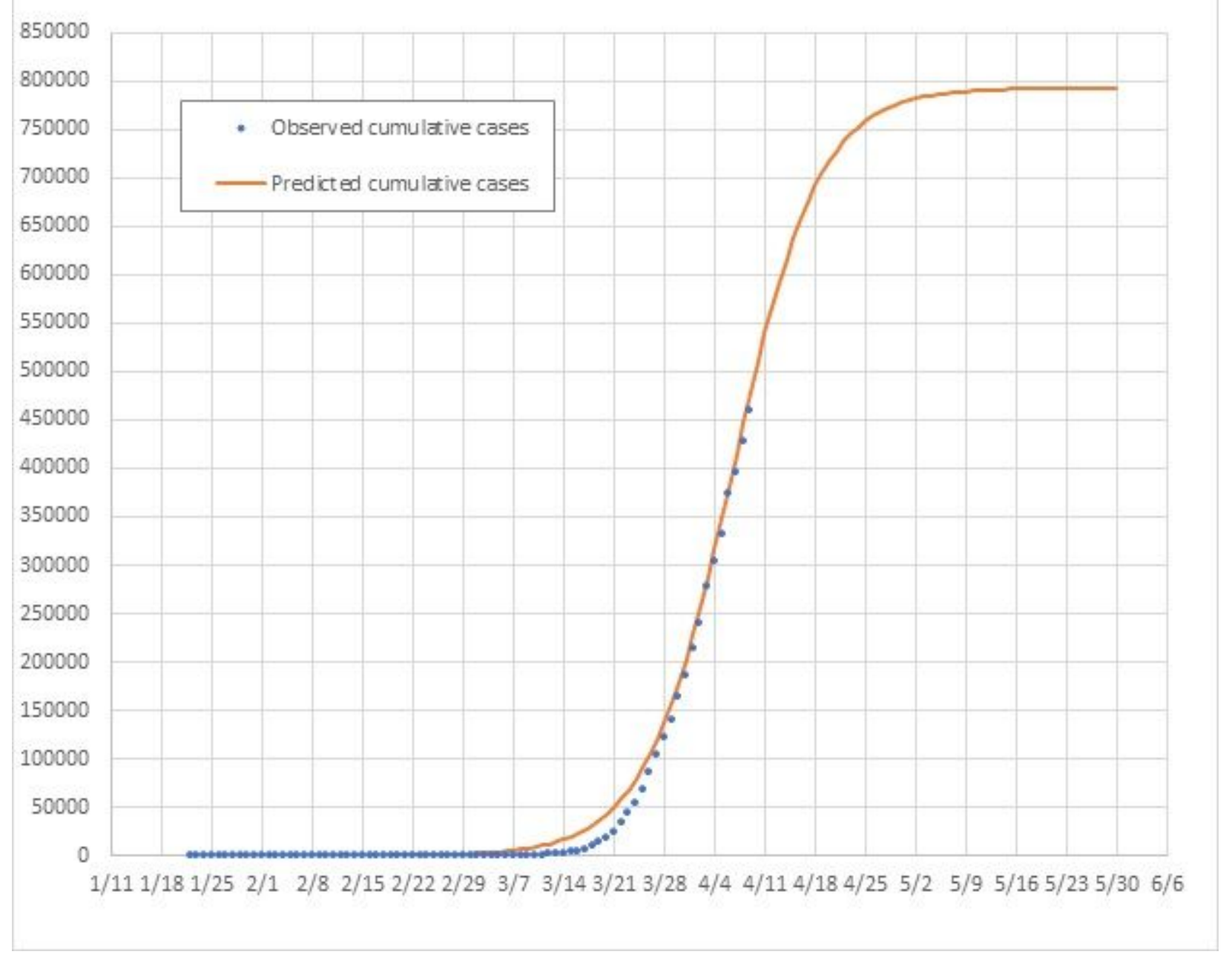

Figure 2

Observed vs. model-estimated and forecasted daily cumulative COVID-19 cases, January 22 - May 30, U.S.A. 